\title{
Subcellular S-factors for low-energy electrons: A comparison of Monte Carlo simulations and continuous-slowing-down calculations
}

\author{
D. EMFIETZOGLOU ${ }^{1}$, K. KOSTARELOS ${ }^{2}$, P. HADJIDOUKAS $^{3}$, C. BOUSIS $^{1}$, \\ A. FOTOPOULOS ${ }^{4}$ A. PATHAK 5 , \& H. NIKJOO ${ }^{6}$ \\ ${ }^{1}$ Medical Physics Laboratory, University of Ioannina Medical School, Ioannina, Greece, ${ }^{2}$ Nanomedicine Laboratory, Centre \\ for Drug Delivery Research, School of Pharmacy, University of London, UK, ${ }^{3}$ Department of Computer Science, University of \\ Ioannina, and ${ }^{4}$ Department of Nuclear Medicine, University of Ioannina Medical School, Ioannina, Greece, ${ }^{5}$ School of \\ Physics, University of Hyderabad, Hyderabad, India, and ${ }^{6}$ Radiation Biophysics Group, Medical Radiation Physics, \\ Karolinska Institute, Stockholm, Sweden
}

(Received 2 February 2008; revised 1 fuly 2008; accepted 16 August 2008)

\begin{abstract}
Purpose: To study the energy deposition by low-energy electrons in submicron tissue-equivalent targets by comparing two widely used methodologies, namely, the continuous-slowing-down-approximation (CSDA) convolution integral and the Monte Carlo (MC) simulation.

Methods: An MC track-structure code that simulates collision-by-collision the complete slowing down process is used to calculate the energy deposition in spherical volumes of unit density water medium. Comparisons are made with calculations based on the CSDA convolution integral using both empirical and MC-based range-energy analytic formulae.

Results: We present self-irradiation absorbed fractions and S-factors for monoenergetic electrons of initial energies from 0.1-10 keV distributed uniformly in spheres of 5,10,50,100,500, and $1000 \mathrm{~nm}$ radius. The MC and CSDA results were found, in some cases, to differ by a factor of 2 or more; differences generally increase with decreasing sphere size. Contrary to high energies, the uncertainties associated with the straight-ahead approximation implicit in the CSDA calculations are of the same order as those related to straggling and $\delta$-ray effects.

Conclusion: The use of the CSDA methodology may be unsuitable for the sub-micron scale where a more realistic description of electron transport becomes important.
\end{abstract}

Keywords: Auger electron, Monte Carlo, cellular dosimetry, targeted radiotherapy, track structure

\section{Introduction}

Any radiotherapeutic modality should aim to the delivery of a lethal radiation dose to all malignant cells while avoiding prohibitive radiation-induced toxicity in healthy tissues. Although this goal may be feasible for some well-localized (bulky) solid tumors, it is less so for disseminated disease which is characterized by single or small clusters of tumor cells. Targeted radiotherapy utilizing electron and/ or alpha particle-emitting radionuclides seem to provide an appealing alternative for micrometastatic and disseminated diseases (Sgouros 1995). It is therefore not surprising that radioimmunotherapy has so far been found most successful in the treatment of blood-related cancers, most notably, non-Hodgkin's lymphoma (NHL) (Goldenberg 2001) where the well-known solid tumor barriers are diminished (Jhanwar and Divgi 2005, Sharkey and Goldenberg 2005). In fact, after almost 30 years of research, the first two radiopharmaceuticals for the treatment of NHL are now in the market $\left(\right.$ Zevalin $^{\mathrm{TM}}$ and Bexxar ${ }^{\mathrm{TM}}$ ) while several more are currently under clinical trials (Goldenberg 2001, Milenic et al. 2004, Brans et al. 2006). Aside from practical issues concerning radiochemistry and pharmacokinetics, the physical characteristics of the radionuclide provide, at least initially,

Correspondence: Dr Hooshang Nikjoo, Radiation Biophysics Group, Medical Radiation Physics, Karolinska Institute, SE17176 Stockholm. Tel: +4685177 5190. E-mail: hooshang.nikjoo@ki.se 
a reasonable starting point for undertaken useful dosimetric calculations for assessing their potential efficacy (Stabin 2006).

At the single-cell level short-range charged particles, such as $\alpha$-particles or Auger electrons, should be more appropriate than the long-range $\beta$ electrons (Wheldon et al. 1991, Hindorf et al. 2005, 2007). Both $\alpha$-particles and Auger electrons present two distinct advantages, namely, the substantial reduction of cross-fire to nearby healthy cells and the occurrence of a densely ionizing energy deposition pattern in matter associated with increased radiobiological effectiveness (Humm et al. 1993, Wheldon 1994, Zweit 1996, Mariani et al. 2000, Kassis 2004, Kassis and Adelstein 2005). However, unlike $\alpha$ particles, Auger electrons are much less radiotoxic to non-targeted (healthy) cells in the blood or bone marrow since, by and large, they are unable to penetrate the cytoplasm and reach the nucleus, generally considered the most radiobiologically important component of the cell. Given their highly localized energy deposition pattern in matter, it is clear that, contrary to the case of $\beta$ electrons, dosimetry at the macroscopic level (e.g., at the organ/tissue level) may be unsuitable for Auger electrons, and tools typical to the fields of microdosimetry and track-structure theory need to be adopted (Humm et al. 1994, O’Donoghue 1999, Bardies and Pihet 2000).

The aim of the present study is to quantify by two different methodologies the energy deposition by low-energy electrons $(0.1-10 \mathrm{keV})$ in tissueequivalent volumes of sub-micron dimensions $(5-1000 \mathrm{~nm})$. Calculations have been carried out by our Monte Carlo (MC) track-structure code which fully accounts for the stochastic nature of electron tracks as well as by the continuous-slowingdown-approximation (CSDA) convolution integral using analytic range-energy formulae. The latter approach has been used by the Medical Internal Radiation Dose (MIRD) Committee (Goddu et al. 1997) for dosimetry at the cellular level $(>1 \mu \mathrm{m})$.

\section{Methodology}

The standard approach for absorbed dose calculations for internally distributed radionuclides makes use of the so-called MIRD schema (Stabin 2006). Within this formalism the (spatial) mean absorbed dose to a target region $r_{k}$ from radioactivity in a source region $r_{h}$ is given by:

$$
\bar{D}\left(r_{k} \leftarrow r_{h}\right)=\tilde{A}_{h} S\left(r_{k} \leftarrow r_{h}\right)
$$

where $\tilde{A}_{h}$ is the number of radionuclide decays that take place within the source region $\mathrm{r}_{\mathrm{h}}$, and $S$ is the absorbed dose in the target region $r_{k}$ per decay in the source region $\mathrm{r}_{\mathrm{h}}$ given by:

$$
S\left(r_{k} \leftarrow r_{h}\right)=\sum_{i} \frac{\Delta_{i} \phi_{i}\left(r_{K} \leftarrow r_{h}\right)}{m_{k}}
$$

where $\phi_{i}\left(r_{k} \leftarrow r_{h}\right)$ is the fraction of energy emitted from the source region that is absorbed in the target region for the $\mathrm{i}$-th radiation component (i.e., type and energy), $\Delta_{\mathrm{i}}$ is the mean energy of the $\mathrm{i}$-th radiation component, and $m_{k}$ is the mass of the target region. Although $\tilde{A}_{h}$ may depend on various biological factors related to the kinetics of the radiopharmaceutical within the body, the S-factor is a purely physical quantity that depends solely on the type and transport properties of the radiation as well as the geometry of the problem. The calculation of S-factors may proceed irrespective of any knowledge of $\tilde{A}_{h}$ Thus, the MIRD Committee has provided extensive tabulations of S-factors and related quantities, such as absorbed fractions and dose-point-kernels (DPK), from the organ down to the cellular level for a variety of radionuclides and monoenergetic photons and electrons (Stabin 2006). For use at the macroscopic level of organs and tissues calculations were first performed by the deterministic transport equation (Spencer 1955, Berger 1971, Siegel and Stabin 1994), and followed by several generalpurpose condensed-history MC codes (Berger 1973, Simpkin and Mackie 1990, Seltzer 1991, Cross et al. 1992, Bolch et al. 1999, Stabin and Konijnenberg 2000, Ferre et al. 2007). A recent comparison revealed that, at the macroscopic level, a detailed-history simulation does not provide any advantage over the much faster condensed-history transport scheme (Cho et al. 2007). Several studies have extended the application of the above macroscopic methods down to the microscopic level of single cells (Jungerman et al. 1984, Bardies et al. 1990, Gardin et al. 1995, Nahum 1996, Faraggi et al. 1998, Hartman et al. 2000, Stewart et al. 2002, Syme et al. 2004).

Condensed-history codes are expected to be increasingly inaccurate as we approach the cellular and especially the sub-cellular level, since the adopted energy cut-off for electron transport (typically about $1-10 \mathrm{keV}$ ) imposes a lowest spatial limit of the order of $0.1-1 \mu \mathrm{m}$. As a result, extending the earlier work of Howell et al. (1989) and Goddu et al. (1994a, 1994b), the MIRD Committee has adopted a simple deterministic approach to calculate cellular S-factors based on the CSDA using an analytic range-energy formula (Goddu et al. 1997). Within this framework the absorbed fraction in 
Equation (2) is obtained by the following convolution integral:

$$
\phi_{i}\left(r_{K} \leftarrow r_{h}\right)=\left.\int \Psi_{r_{k} \leftarrow r_{h}}(x) \frac{1}{E_{i}} \frac{d E}{d X}\right|_{X\left(E_{i}\right)-x} d x
$$

where $\Psi_{r_{k}} \leftarrow r_{h}(x)$ is the geometric reduction factor representing the mean probability that a randomly directed vector of length $x$ that starts from a random point within the source region ends within the target region (Goddu et al. 1997), and $d E /\left.d X\right|_{X\left(E_{i}\right)-x}$ is an 'effective' stopping power evaluated at $X\left(E_{i}\right)-x$ which is the residual range of a particle with initial energy $E_{\mathrm{i}}$ after passing (a linear) distance $x$ through the medium. Note that, contrary to the organ level, the contribution of photons to cellular S-factors may be safely neglected. In essence, Equation (3) is a convolution between a geometric term that characterizes the source distribution in the medium and an energy-loss term that defines the relevant DPK. The stopping power $d E / d X$ was evaluated by the MIRD Committee on the basis of the Howell et al. (1989) expressions which represent a low-energy improvement to Cole's original range-energy formula (Cole 1969). This computational scheme assumes that the energy-loss (potential and kinetic energy) experienced by the primary particle in inelastic collisions is deposited at a continuous rate along a straight-line trajectory. Also important for the present context is that the CSDA assigns zero range to all secondary electrons.

However, the extension of the range of applicability of the CSDA convolution integral from the cellular $(>1 \mu \mathrm{m})$ to the sub-cellular (nm) level may be questioned on the grounds that at the submicron scale the 'discrete' nature of interactions resulting in energy-loss straggling, angular deflections, and secondary electron production (hereafter called $\delta$-ray range) cannot be neglected. Monte Carlo track-structure codes simulating collision-bycollision the slowing down process of all generations of particles are known to be best suited for such applications since they methodologically account for all the above deficiencies of the CSDA (Nikjoo et al. 2006). The (micro) dosimetry of low-energy Auger electrons at the sub-cellular (and DNA) level have been studied by several track-structure codes (Charlton 1986, Wright et al. 1990, Pomplun 1991, Bolch and Kim 1994, Nikjoo et al. 1996, Ftacnikova and Bohm 2000a, 2000b, Torres-Garcia et al. 2006).

In the present study an attempt is made to quantitatively assess the energy deposition of lowenergy electrons in sub-micron tissue-equivalent volumes $(<1 \mu \mathrm{m})$. By comparing CSDA calculations using different range-energy expressions (i.e., empirical vs. MC-based) against direct MC simula- tions, we examine both the limits of applicability of the CSDA method and the effect of its various assumptions such as, the straight-ahead approximation, the continuous energy-loss rate and the neglect of $\delta$-ray range. The track-structure code used in the present work is an updated version of our MC4V code (Emfietzoglou et al. 2000a, 2000b) which simulates stochastic tracks of electrons in a unit density (and homogeneous) water-vapor medium. The present version of the code includes an improved model for the total and differential ionization cross-sections which results in better agreement with the recent recommendations of Itikawa and Mason (2005). This development also brings our collision stopping power at the $1-10 \mathrm{keV}$ range to within $3 \%$ of the respective NIST/ICRU values which have a reported uncertainty of $3-10 \%$ in this range. Also, following Kim (1972) and Wilson and Nikjoo (1999), the Grosswendt and Waibel (1978) scheme for modeling the differential-in-angle ionization cross-section has now been substituted by the appropriate Bethe asymptotic formula using the experimental optical-oscillator-strength of water. This development substantially improves the agreement with the available experimental data on the ejection angle of low-energy secondaries. Finally, the Grosswendt and Waibel (1978) screening parameter in the elastic cross-sections (i.e., the screened Rutherford formula) which had been established for nitrogen has now been substituted by the value provided by Uehara et al. (1992) which was based on water data.

The simulation results presented are average values derived from over 100,000 primary electron histories. The overall (statistical) uncertainty is estimated to be less than $1 \%$. The primary electron energies cover the range from $0.1-1 \mathrm{keV}$ with a step of $0.1 \mathrm{keV}$ and from $1-10 \mathrm{keV}$ with a step of $1 \mathrm{keV}$. The electron cut-off was set at $1 \mathrm{Ry}(=13.6 \mathrm{eV})$ due to limitations of our inelastic model at lower energies. We consider sphere volumes of $5,10,50$, 100,500 , and $1000 \mathrm{~nm}$ in radius which are relevant to critical sub-cellular targets ranging from the DNA to the cell nucleus level. To the extent of overlap with some of the results of Emfietzoglou et al. (2007) on the same subject, the present simulation results should be considered more accurate and update our previously published values which had been based on the original version of the MC4V code (Emfietzoglou et al. 2000a, 2000b) and had also a somewhat larger statistical uncertainty due to the smaller number of histories used.

\section{Results}

Figure 1 (panel a) presents our MC-calculated range of monoenergetic electrons in a unit density 

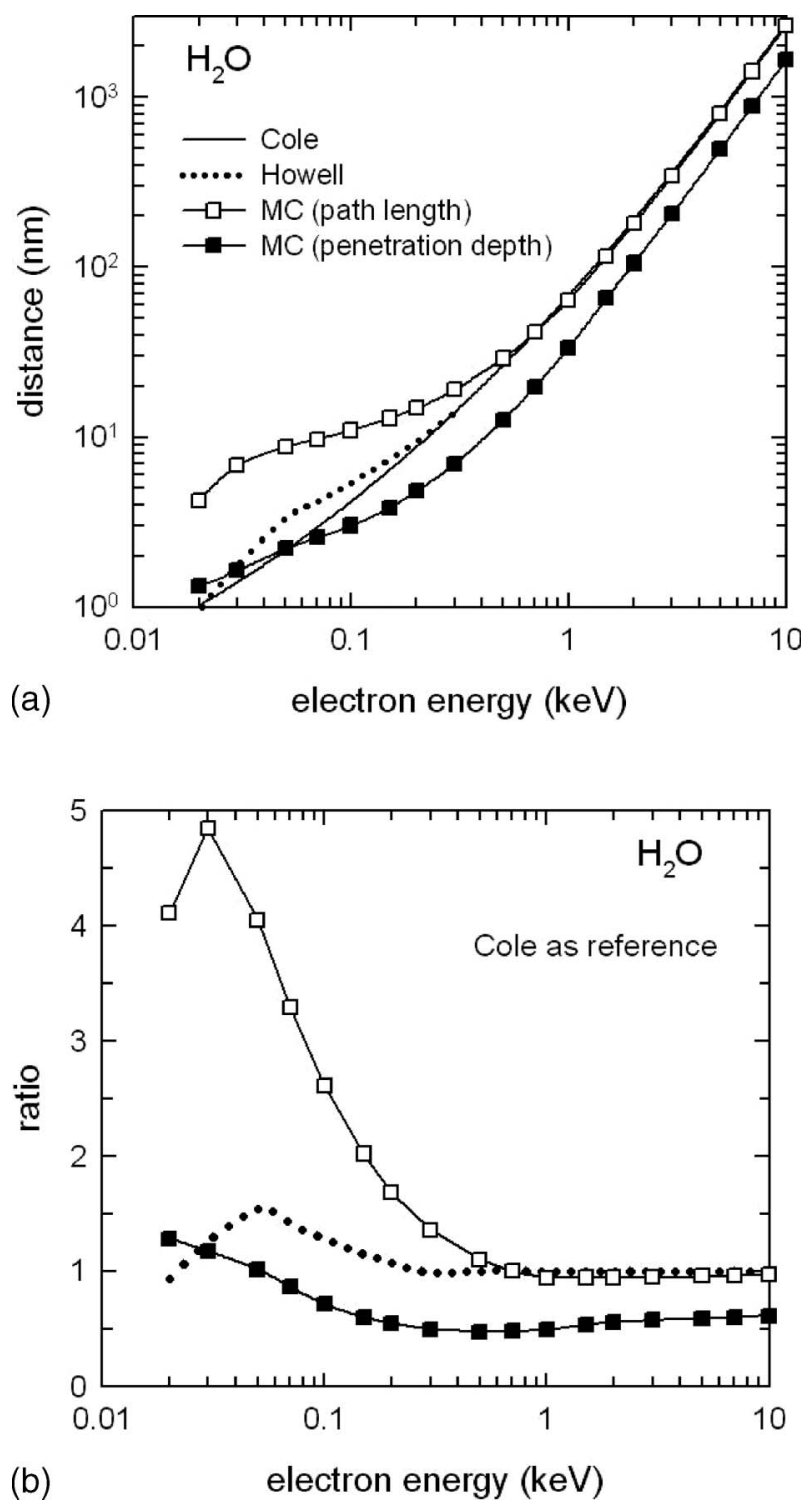

Figure 1. (a) Various measures of electron 'range' in a unit density water medium calculated by the analytic formulae of Cole (1969) and Howell et al. (1989) as well as by our Monte Carlo track structure code. (b) The ratio of the Howell et al. and Monte Carlo data depicted in panel a against the Coles values.

water-vapor medium along with the predictions of the Cole (1969) and Howell et al. (1989) analytic formulae. The latter is essentially a low energy empirical improvement $(<400 \mathrm{eV})$ of Cole's original formula. The designation 'path' denotes the mean length of the tortuous electron track whereas the designation 'penetration' denotes the mean length of the straight-line between the initial and most distant interaction point of the track. Thus, the path length is most closely related to the CSDA-range, i.e. the integral over the reciprocal of the stopping power. On the other hand, the penetration-depth relates to the transmission probability for a point source in a spherical geometry. In panel $b$ we provide the ratio of the various measures of 'range' depicted in panel a over that of Cole's.

Self-irradiation absorbed fractions for monoenergetic electrons distributed uniformly in spheres of different size are depicted in Figure 2. The results have been obtained by both the CSDA convolution integral of Equation 3 (here $r_{k}=r_{h}, i=1$ ) using the Cole or Howell et al. range-energy formulae and by direct MC simulation. For the latter a homogeneous and infinite water-vapor medium at unitdensity were assumed, while the target spheres were subdivided into scoring shells of $0.5 \mathrm{~nm}$ thickness. The point of origin of each electron was uniformly randomized inside the sphere according to the volume mass to mimic a uniform activity distribution.

To further study the effect of the straight-ahead approximation implicit in the CSDA calculations, we have fitted in Figure 3 the MC-calculated pathlength (panel a) and penetration-depth (panel b) by analytic functions of the form $R=\mathrm{a}(b+c T)^{d}$ where $R$ is the range in $\mathrm{nm}$ and $T$ the energy in $\mathrm{keV}$. The values of the coefficients for the path-length (penetration-depth) data are: $a=8.421 \times 10^{-6}$ $\left(7.511 \times 10^{-6}\right), \quad b=17380 \quad(800.1), \quad c=5036$ (4702), $d=1.801$ (1.783). These analytic MC-based range-energy formulae may then be used through Equations (2) and (3) for absorbed fraction and Sfactor calculations within the CSDA methodology.

In Figure 4 we present $S$-factors for a uniform distribution of activity in sub-micron spherical volumes. A comparison is made between CSDA calculations using various range-energy expressions and direct MC simulations. The S-factor difference (in \%) among the various calculations and the CSDA-Cole values are depicted in Figure 5. The large decrease of S-factors (by almost an order of magnitude) with increasing sphere size is largely due to the inverse proportionality of the absorbed dose with the target mass (Equation 2) which, in turn, is proportional to the third power of the radius; the absorbed fraction differences between the spheres are much smaller (see Figure 2).

\section{Discussion}

The aim of the present study is to quantitatively assess the energy deposition of low-energy electrons in sub-micron tissue-equivalent volumes by both the CSDA convolution integral which has been extensively used at the cellular and multicellular level as well as by an MC track-structure code which is a standard microdosimetric tool. The former method has also been adopted by the MIRD Committee for calculating cellular S-factors for spherical volumes of $1-10 \mu \mathrm{m}$ radius (Goddu et al. 1997). By comparing CSDA calculations based on different range-energy 


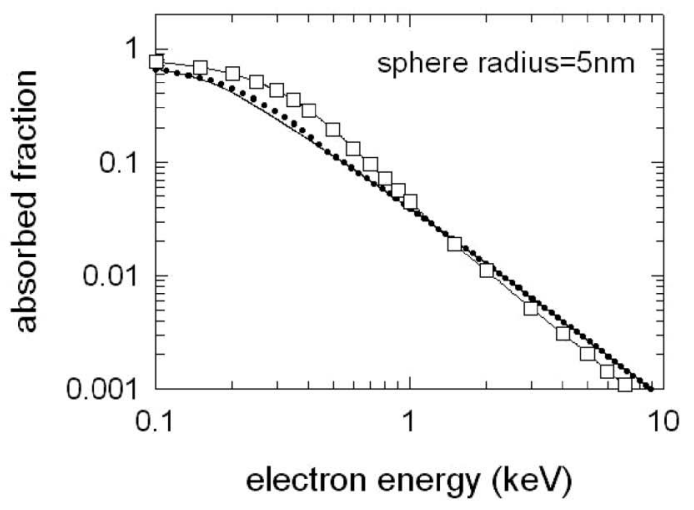

(a)

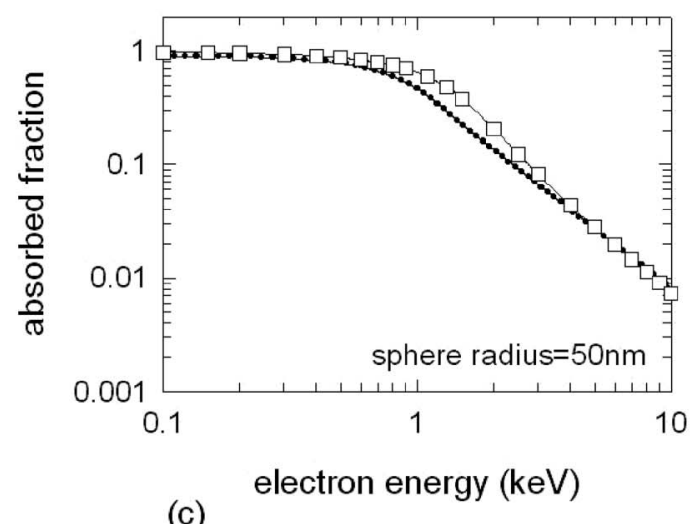

(c)

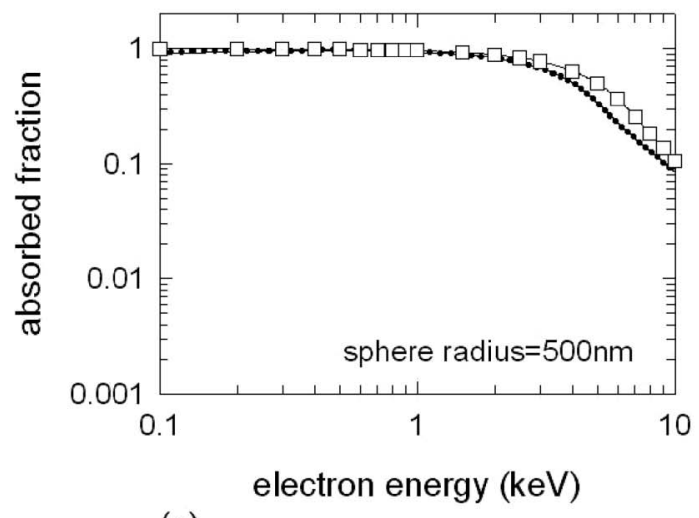

(e)

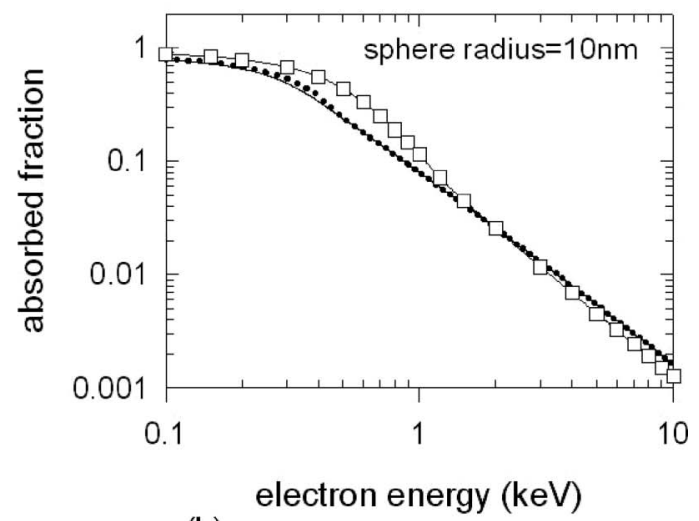

(b)
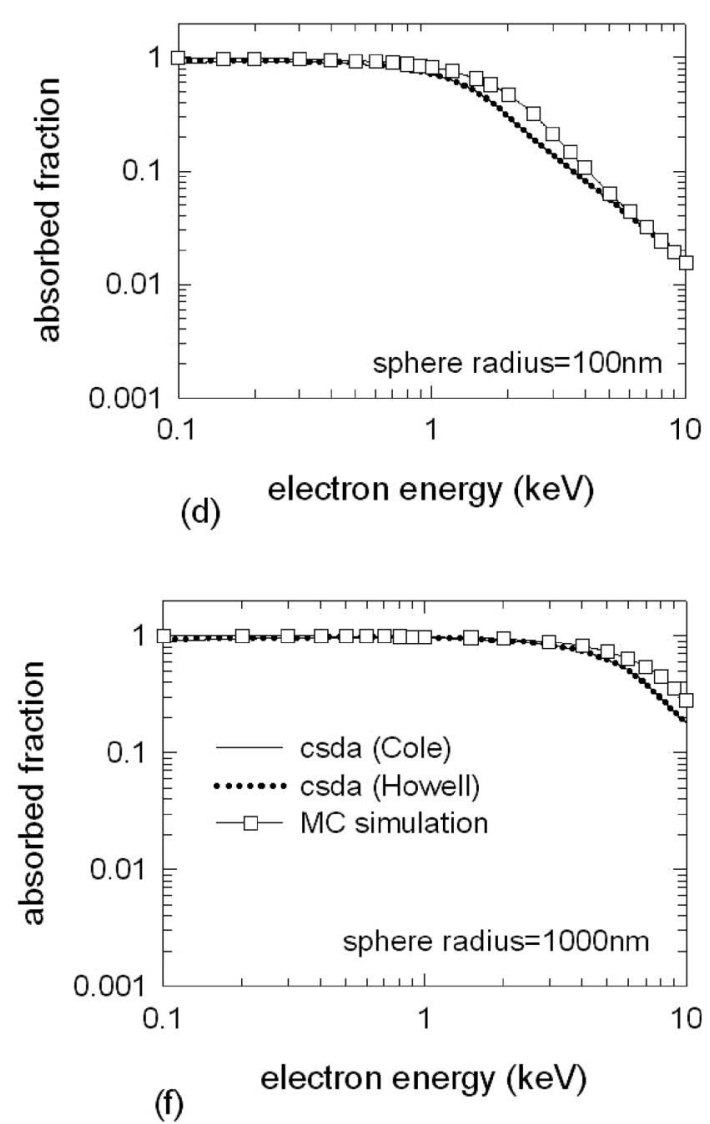

Figure 2. Self-irradiation absorbed energy fractions for a uniform distribution of monoenergetic electrons as a function of their initial energy for spherical volumes of various sizes. The $\mathrm{MC}$ data correspond to track-structure simulation results.

expressions (i.e., empirical versus $\mathrm{MC}$-based) as well as against direct MC simulations, we examine both the limits of applicability of the CSDA method and the effect of its various assumptions, such as the straight-ahead approximation, the continuous energy-loss rate and the neglect of $\delta$-ray range.

It may be clearly seen from Figure 1 that from $10 \mathrm{keV}$ down to about $500 \mathrm{eV}$ the analytic formulae of Cole and Howell et al. are in better agreement with the MC-path-length than the MC-penetrationdepth data. The difference between the Cole and
MC-penetration-depth results is up to $\sim 50 \%$ (panel b). The Howell et al. curve, for the most part, is further shifted towards the MC-path-length. However, the effective stopping power to be used under the straight-ahead approximation should formally reflect the energy loss per unit distance across the penetration-depth and not across the pathlength. This is because the integral in Equation (3) is over a (linear) distance across the medium $(\mathrm{d} x)$ and not a distance along the particle track; thus, $X$ should be a measure of the penetration-depth and 

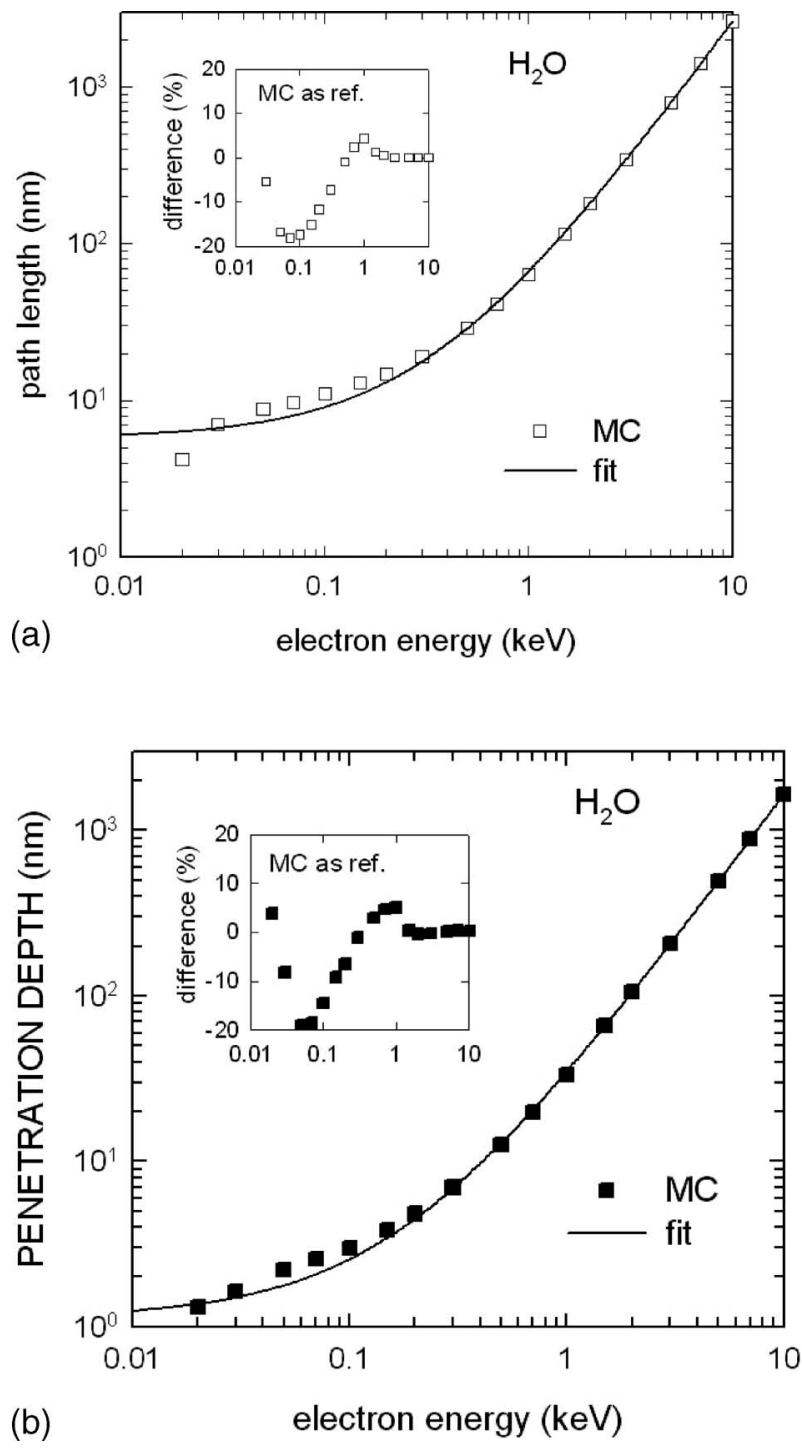

Figure 3. Analytic fitting of the Monte Carlo based path-lengths (a) and penetration-depths (b). The insets depict the percentage difference between the fitting and the data.

not the path-length. It follows from Figure 1 that, under the straight-ahead approximation, the Cole or Howell et al. effective stopping power will underestimate the energy loss calculated by the MC simulations.

The above finding is confirmed in Figure 2 where the absorbed fractions obtained from MC simulations are higher than the CSDA (Cole/Howell et al.) values at electron energies where the penetration range is comparable to the sphere size. The situation is reversed at sufficiently high energies where the $\delta$ rays may become capable of escaping the target volume; the neglect of the finite $\delta$-ray range in the CSDA method will then overestimate the absorbed fractions. The above effects are most clearly seen in the case of the 5 and $10 \mathrm{~nm}$ spheres where, due to the straight-ahead approximation, the MC simulation curves start above the CSDA ones while, gradually, fall below for energies higher than a few $\mathrm{keV}$ due to the $\delta$-ray range. The latter effect is not observed for the larger spheres because the maximum electron energy examined $(10 \mathrm{keV})$ is not high enough compared to the sphere size. As expected, differences between the absorbed fractions obtained by the use of either the Cole or Howell et al. formula gradually vanish with increasing sphere size. Differences are noticeable only for the 5 and $10 \mathrm{~nm}$ spheres since the Howell et al. formula departs significantly from the original Cole formula below about $200 \mathrm{eV}$ where the residual range of electrons is less than $\sim 10 \mathrm{~nm}$.

In the present work, S-factor calculations are based on the equation: $S(r)=E_{0} \times \Phi(r)$ where $\Phi(r)$ is the specific absorbed fraction in region $r$ and $\mathrm{E}_{0}$ the initial electron energy. Thus, it is straightforward to convert the results of Figure 2 to S-factor profiles. To gain some further insight into the CSDA versus MC differences, we have provided in Figure 3 analytic fitting functions for MC-based electron path-lengths and penetration-depths to be used in CSDA calculations. These formulae along with the previous methodologies have been used to generate the $S$-factor profiles depicted in Figures 4 and 5. Thus, differences between the CSDA results obtained by the MC-based range-energy formulae for pathlength and penetration-depth will be solely due to the effect of angular deflections of the primary electron (straight-ahead approximation). Additionally, differences between the CSDA method using MC-based penetration-depth and direct MC simulation will strictly reflect the effect of straggling and $\delta$-rays, i.e., the straight-ahead approximation is not involved.

Several conclusions may be drawn from the results of Figures 4 and 5. Firstly, a comparison between the MC simulation and CSDA-Cole/Howell et al. results shows that, with increasing sphere size, differences decrease and shift at higher energies. The maximum difference ranges from $\sim 60 \%(1 \mu \mathrm{m}$ sphere) to $\sim 80 \%$ ( $5 \mathrm{~nm}$ sphere). It is worth pointing out that these differences are noticeably larger than the ones reported in Emfietzoglou et al. (2007) which, as stated above, are based on an earlier version of the code. As in the case with the absorbed fraction calculations, the maximum differences are reached when the electron penetration-depth becomes comparable to the sphere size and they are attributable to the overestimation of the electron penetration-depth by the Cole/Howell et al. formulae. In contrast, at high energies, the CSDA-Cole/ Howell et al. progressively overestimate the MC simulations by up to $30 \%$ ( $5 \mathrm{~nm}$ sphere) due to the finite range of $\delta$-rays. Secondly, differences between results obtained from direct $\mathrm{MC}$ simulation and 


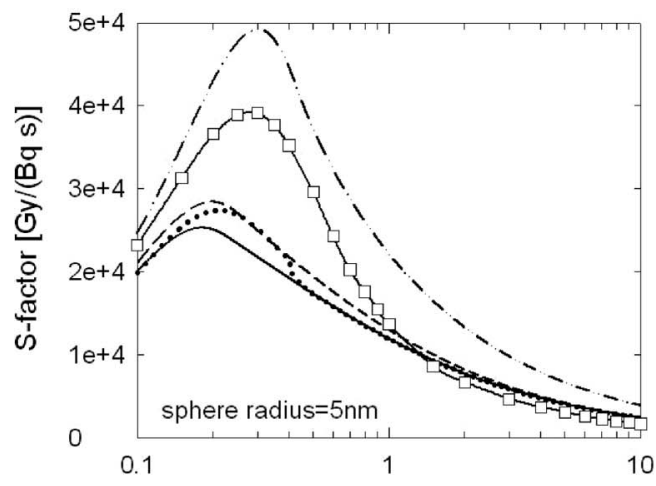

(a) electron energy (keV)
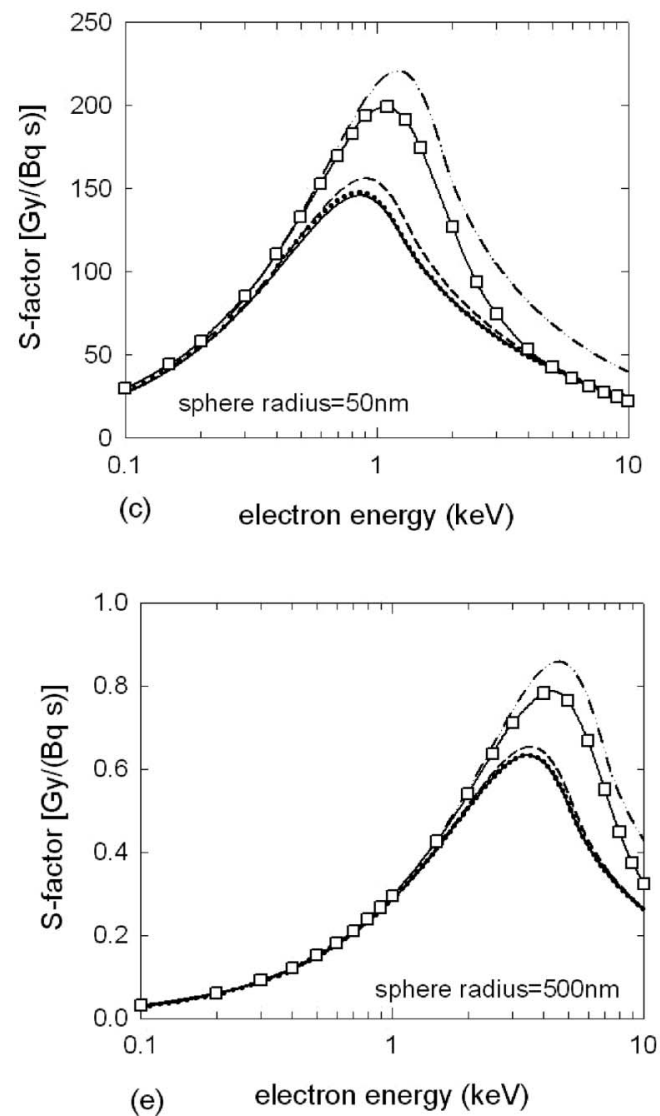

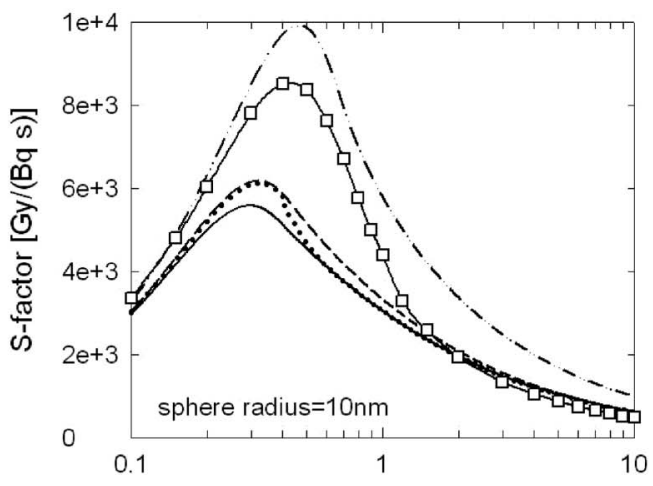

(b) electron energy (keV)
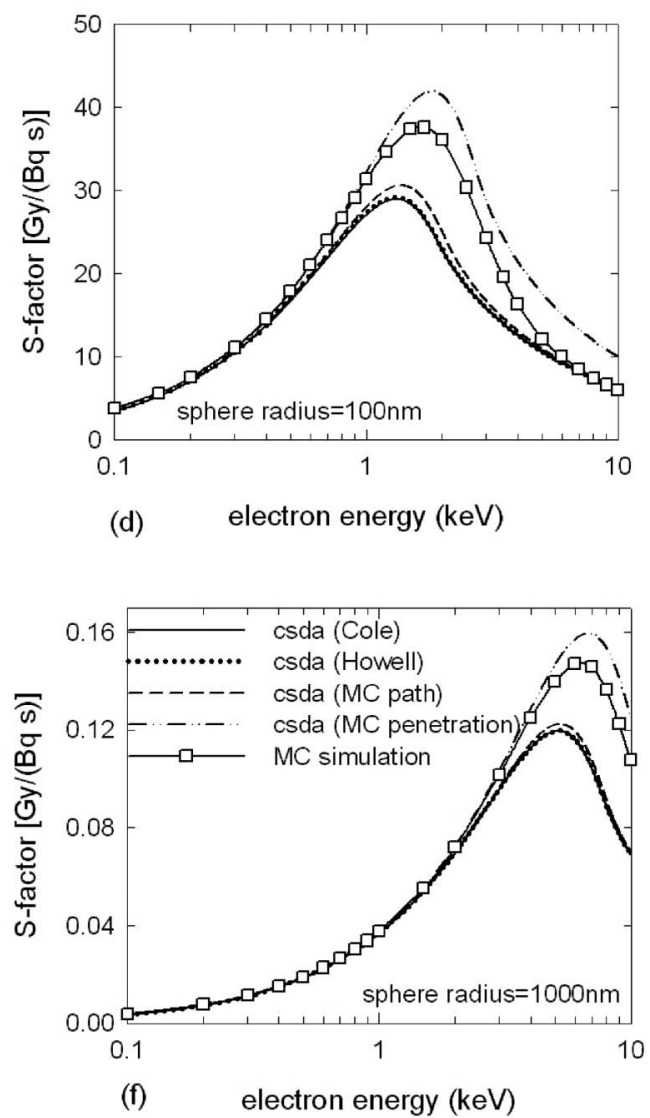

Figure 4. S-factors for a uniform distribution of monoenergetic electrons as a function of their initial energy for spherical volumes of various sizes. The MC data correspond to track-structure simulation results. The CSDA data denoted as 'MC path' and 'MC penetration' correspond to analytic calculations by the convolution integral method (Equations 2 and 3) using range-energy expressions for the pathlength and penetration-depth, respectively, derived from Monte Carlo simulations.

CSDA-penetration-depth calculations increase with electron energy and decrease with sphere size. These observations are compatible with the fact that both straggling and $\delta$-rays are more pronounced at high electron energies and small spheres due, respectively, to the fewer number of collisions per unit distance and the larger fraction of initial energy carried outside the sphere by $\delta$-rays. Ignoring these effects results in an overestimation of the S-factor which, at $10 \mathrm{keV}$ electron energy, ranges from $20 \%(1 \mu \mathrm{m}$ sphere) to $130 \%$ (5 nm sphere). Thirdly, a comparison between CSDA calculations based on the MCbased path-length and penetration-depth shows that the latter are higher by up to $80 \%$ for the $1 \mu \mathrm{m}$ sphere and by up to $100 \%$ for the $5 \mathrm{~nm}$ sphere due to corresponding differences in the energy-loss rate across the medium. Under conditions of nearcomplete absorption (sphere sufficiently large or electron energy sufficiently small) the straightahead approximation is, obviously, inconsequential. 


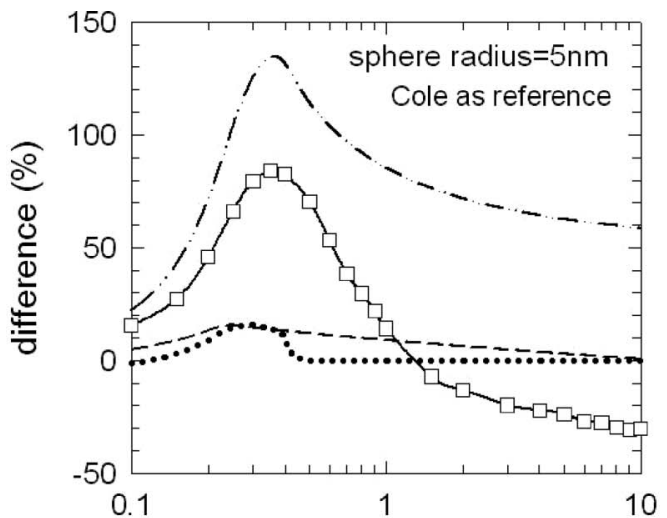

(a) electron energy (keV)
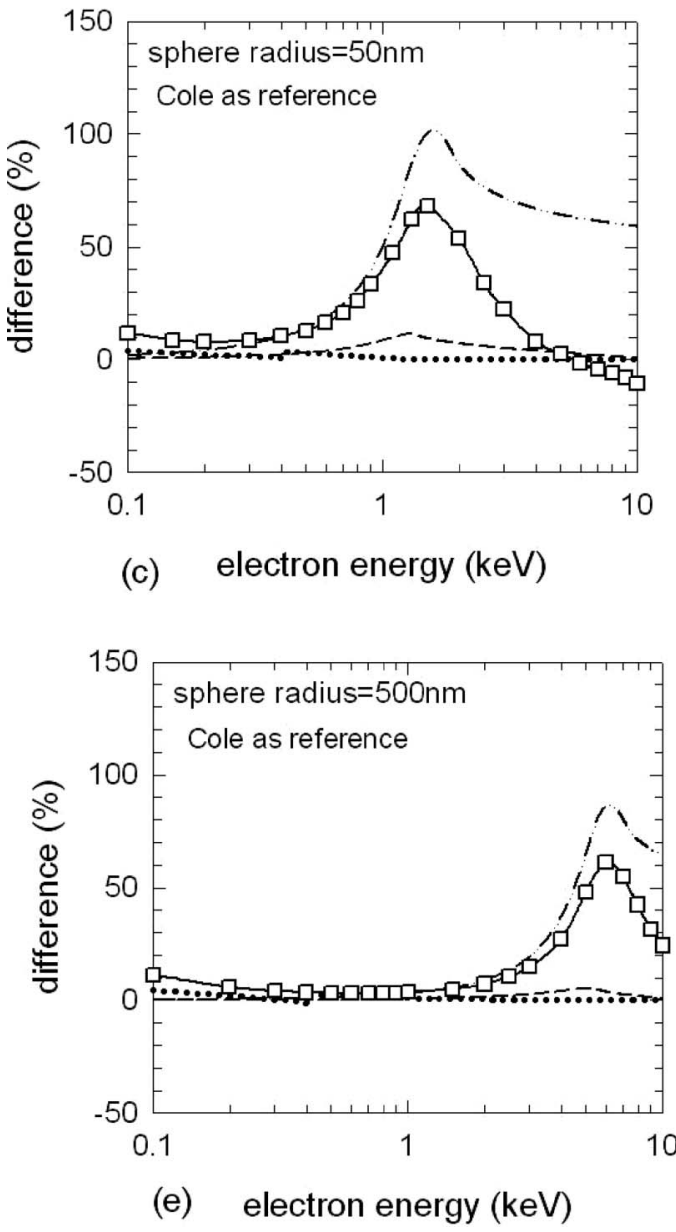

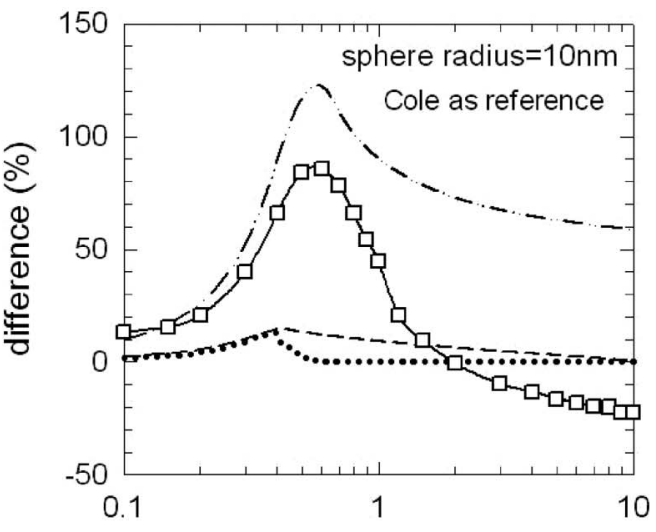

(b) electron energy (keV)
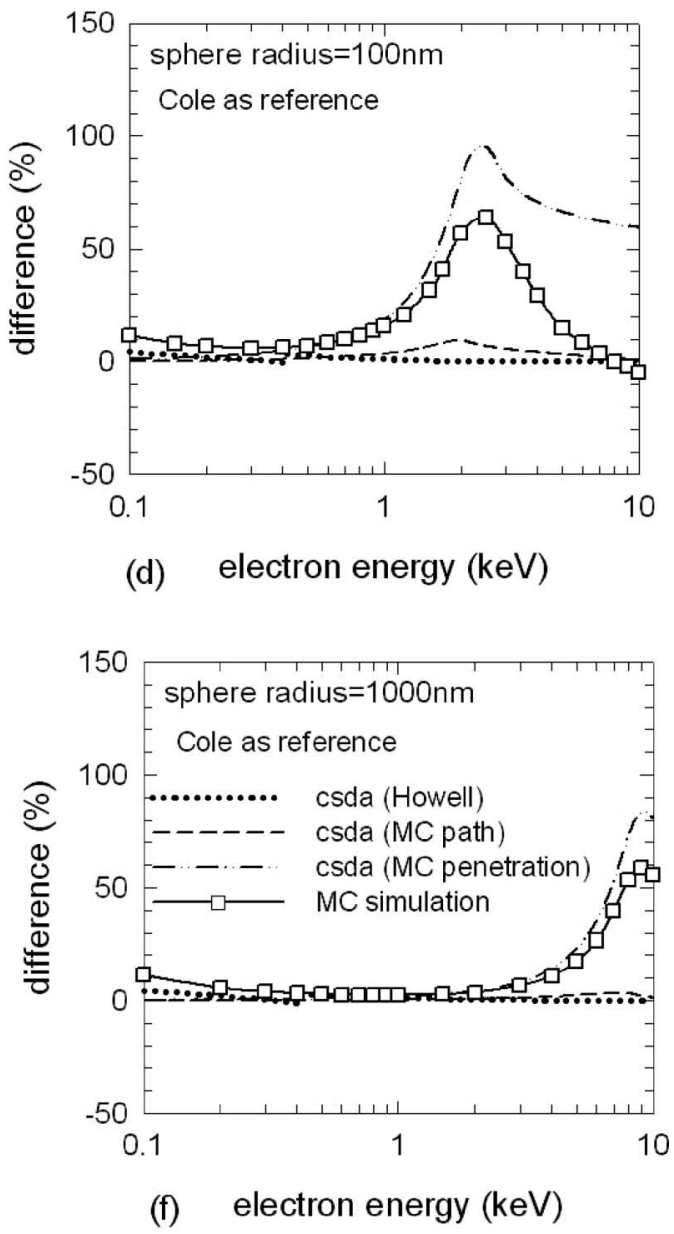

Figure 5. The S-factor difference of the results presented in Figure 4 with the CSDA-Cole values used as reference.

The maximum differences reported above take place when the sphere size is comparable to the electron penetration distance since, in such cases, a small variation in the diffusion capability of electrons (path-length versus penetration-depth) will have the most impact in the amount of energy imparted within the sphere. Finally, as also noted earlier in relation to Figure 2, the use of the Howell et al. formula results in a noticeable difference from the results obtained by Cole's formula only for the 5 and $10 \mathrm{~nm}$ spheres where maximum deviations of 10 $15 \%$ are observed. With increasing sphere size the maximum difference falls below $5 \%$.

Regarding the reliability of the MC results, it should be borne in mind that the cross-section input is a critical component in any MC code. Judging from the 
overall agreement of the present set of cross-sections with available experimental data on the elastic and inelastic scattering of electrons in water vapor, it is concluded that our simulations should be accurate to better than $10-20 \%$. In fact, as a rule-of- thumb a $5-10 \%$ uncertainty is generally associated with the experimental cross-section measurements. The above level of uncertainty does not invalidate any of the conclusions of the current study. However, the accuracy of the present MC simulations is also limited by the degree of which the gas-phase approximation is valid in our case. Several studies have shown that for low-energy electrons and nanometer-size volumes condensed-phase effects reflecting differences between the vapor and liquid water crosssections may become significant (LaVerne and Mozumder 1986, Paretzke et al. 1986, Nikjoo et al. 1994, Uehara et al. 1999, Emfietzoglou et al. 2005a). Recently, an improved set of inelastic and stopping cross-sections specific for liquid water has been developed based on a semi-empirical dielectricresponse-function which accurately represents the available experimental data (Emfietzoglou and Nikjoo 2005b, 2007, Emfietzoglou et al. 2005). Given that cells are in a liquid/solid-like phase, it is envisioned that the use of this new set of cross-section data will permit more realistic electron transport simulations at the nano- and micro-scale.

\section{Conclusion}

Absorbed fraction and S-factor calculations for lowenergy electrons in the $0.1-10 \mathrm{keV}$ energy range uniformly distributed in spheres of 5-1000 nm radius showed that the CSDA convolution integral may, in some cases, differ from direct MC simulation results by a factor of 2 or more. In particular, the neglect of straggling and $\delta$-ray range, inherent in the CSDA methodology, may have a significant effect in the sub-micron scale where the number of interactions is limited and secondary electrons may escape the target volume. The straight-ahead approximation implicit in the CSDA method may also increase the uncertainty of the results since the choice of the electron 'range' to be used in the calculation becomes critical. Although the discrepancies generally increase with decreasing sphere size, it is worth noting that even for the largest sphere examined $(1 \mu \mathrm{m})$ differences between CSDA calculations and direct MC simulations at the $50 \%$ level may be observed. The present results seem to question the adequacy of the CSDA approach at the sub-cellular level where one expects accounting for the discrete nature of interactions by the MC method should lead to more accurate estimates of energy deposition. To that end, the continuous improvement of the cross-section input in the MC codes should be of high priority in order to reduce the systematic uncertainties in the simulations. In particular, a realistic account of the condensed-phase cellular environment in terms of material-specific crosssections (pertinent to liquid/solid water) will be an important step towards more accurate electron transport simulations at this spatial scale.

\section{Acknowledgement}

This work was partly supported by the European Union FP7 ANTICARB (HEALTH-F2-2008201587) research programme.

Declaration of interest: The authors report no conflicts of interest. The authors alone are responsible for the content and writing of the paper.

\section{References}

Bardies M, Lame J, Myers MJ, Simoen JP. 1990. A simplified approach to beta dosimetry for small spheres labelled on the surface. Physics in Medicine and Biology 35:1039-1050.

Bardies M, Pihet P. 2000. Dosimetry and microdosimetry of targeted radiotherapy. Current Pharmaceutical Design 6: 1469-1502.

Berger MJ. 1971. MIRD Pamphlet no. 7: Distribution of absorbed doses around point sources of electrons and beta particles in water and other media. The Journal of Nuclear Medicine 12: 5-23.

Berger MJ. 1973. Improved point kernels for electrons and betaray dosimetry NBSIR-73-107. Gaithersburg MD, National Bureau of Standards.

Bolch WE, Bouchet LG, Robertson JS, Wessels BW, Siegel JA, Howell RW, Erdi AK, Aydogan B, Costes S, Watson EE. 1999. MIRD Pamphlet No. 17: The dosimetry of nonuniform activity distributions-radionuclide $S$ values at the voxel level. The Journal of Nuclear Medicine 40:11S-36.

Bolch WE, Kim E-H. 1994. Calculations of electron single event distributions for use in internal beta microdosimetry. Radiation Protection Dosimetry 52:77-80.

Brans B, Linden O, Giammarile F, Tennvall J, Punt C. 2006. Clinical applications of newer radionuclide therapies. European Journal of Cancer 42:994-1003.

Charlton DE. 1986. The range of high LET effects from I-125 decays. Radiation Research 107:163-171.

Cho SH, Vassiliev ON, Horton JL. 2007. Comparison between an event-by-event Monte Carlo code, NOREC, and ETRAN for electron scaled point kernels between $20 \mathrm{keV}$ and $1 \mathrm{MeV}$. Radiation and Environmental Biophysics 46:77-83.

Cole A. 1969. Absorption of $20-\mathrm{eV}$ to $50,000-\mathrm{eV}$ electron beams in air and plastic. Radiation Research 38:7-33.

Cross WG, Freedman NO, Wong PY. 1992. Beta-ray dose distributions from point sources in an infinite water medium. Health Physics 63:160-171.

Emfietzoglou D, Bousis C, Hindorf C, Fotopoulos A, Pathak A, Kostarelos K. 2007. A Monte-Carlo study of energy deposition at the sub-cellular level for application to targeted radionuclide therapy with low-energy electron emitters. Nuclear Instruments and Methods in Physics Research B: Beam Interactions with Materials \& Atoms 256:547-553.

Emfietzoglou D, Cucinotta FA, Nikjoo H. 2005b. A complete dielectric response model for liquid water: A solution of the Bethe ridge problem. Radiation Research 164:202-211. 
Emfietzoglou D, Karava K, Papamichael G, Moscovitch M. 2003. Monte Carlo simulation of the energy loss of low-energy electrons in liquid water. Physics in Medicine and Biology 48: 2355-2371.

Emfietzoglou D, Nikjoo H. 2005. The effect of model approximations on single-collision distributions of low-energy electrons in liquid water. Radiation Research 163:98-111.

Emfietzoglou D, Nikjoo H. 2007. Accurate electron inelastic cross sections and stopping powers for liquid water over the $0.1-10 \mathrm{keV}$ range based on an improved dielectric description of the Bethe surface. Radiation Research 167: 110-120.

Emfietzoglou D, Papamichael G, Androulidakis I, Karava K, Kostarelos K, Pathak A, Moscovitch M. 2005a. A MonteCarlo study of sub-keV electron transport in water: the influence of the condensed phase. Nuclear Instruments and Methods in Physics Research B: Beam Interactions with Materials \& Atoms 228:341-348.

Emfietzoglou D, Papamichael G, Kostarelos K, Moscovitch M. 2000b. A Monte Carlo track structure code for electrons $(10 \mathrm{eV}-10 \mathrm{keV})$ and protons $(0.3-10 \mathrm{MeV})$ in water: Partitioning of energy and collisions events. Physics in Medicine and Biology 45:3171-3194.

Emfietzoglou D, Papamichael G, Moscovitch M. 2000a. An eventby-event computer simulation of interactions of energetic charged particles and all their secondary electrons in water. Journal of Physics D: Applied Physics 33:932-944.

Faraggi M, Gardin I, Stievenart J-L, Bok BD, Le Guludec D. 1998. Comparison of cellular and conventional dosimetry in assessing self-dose and cross-dose delivered to the cell nucleus by electron emissions of ${ }^{99 \mathrm{~m}} \mathrm{TC},{ }^{123} \mathrm{I},{ }^{111} \mathrm{I},{ }^{67} \mathrm{Ga},{ }^{201} \mathrm{Tl}$. European Journal of Nuclear Medicine 25:205-214.

Ferre L, Chouin N, Bitar A, Lisbona A, Bardies M. 2007. Implementing dosimetry in GATE: dose-point kernel validation with GEANT4 4.8.1. Cancer Biotherapy \& Radiopharmaceutical 22:125-129.

Ftacnikova S, Bohm R. 2000a. Monte Carlo calculations of energy deposition in DNA for Auger emitters. Radiation Protection Dosimetry 92:269-278.

Ftacnikova S, Bohm R. 2000b. Monte Carlo calculations of energy deposition on cellular, multicellular and organ level for Auger emitters. Radiation Protection Dosimetry 92: 279-288.

Gardin I, Faraggi M, Hue E, Bok BD. 1995. Modelling of the relationship between cell dimensions and mean electron dose delivered to the cell nucleus: Application to five radionuclides used in nuclear medicine. Physics in Medicine and Biology 40: 1001-1014.

Goddu SM, Howell RG, Bouchet LG, Bolch WE, Rao DV. 1997. MIRD cellular $S$ values. Reston VA, Society of Nuclear Medicine.

Goddu SM, Howell RW, Rao DV. 1994a. Cellular dosimetry: Absorbed fractions for monoenergetic electron and alpha particle sources and S-factors for radionuclides uniformly distributed in different cell compartments. The Journal of Nuclear Medicine 35:303-316.

Goddu SM, Rao DV, Howell RW. 1994b. Multicellular dosimetry for micrometastases: Dependence of self-dose versus crossdose to cell nuclei on type and energy of radiation and subcellular distribution of radionuclides. The Journal of Nuclear Medicine 35:521-530.

Goldenberg D. 2001. The role of radiolabeled antibodies in the treatment of non-Hodgkin's lymphoma: The coming of age of radioimmunotherapy. Critical Reviews in Oncology/ Hematology 39:195-201.

Grosswendt B, Waibel E. 1978. Transport of low energy electrons in nitrogen and air. Nuclear Instruments and Methods 155: $145-156$.
Hartman T, Lundqvist H, Westlin J-E, Carlsson J. 2000. Radiation doses to the cell nucleus in single cells and cells in micrometastases in targeted therapy with 131I labeled ligands or antibodies. International Journal of Radiation Oncology, Biology, Physics 46:1025-1036.

Hindorf C, Emfietzoglou D, Linden O, Bousis C, Fotopoulos A, Kostarelos K, Flux G. 2007. Single cell dosimetry for radioimmunotherapy of B-cell lymphoma patients with special reference to leukaemic spread. Cancer Biotherapy \& Radiopharmaceuticals 22:357-366.

Hindorf C, Emfietzoglou D, Linden O, Kostarelos K, Strand S-E. 2005. Internal microdosimetry for single cells in radioimmunotherapy of B-cell lymphoma. Cancer Biotherapy \& Radiopharmaceuticals 20:224-230.

Howell RW, Rao DV, Sastry KSR. 1989. Macroscopic dosimetry for radioimmunotherapy: nonuniform activity distributions in solid tumors. Medical Physics 16:66-74.

Humm JL, Howell RW, Rao DV. 1994. Dosimetry of Augerelectron-emitting radionuclides: Report no. 3 of AAPM Nuclear Medicine Task Group No. 6. Medical Physics 21:1901-1915.

Humm JL, Roeske JC, Fisher DR, Chen GTY. 1993. Microdosimetry concepts in radioimmunotherapy. Medical Physics 20:535-541.

Itikawa Y, Mason N. 2005. Cross sections for electron collisions with water molecules Journal of Physical and Chemical Reference Data 34:1-22.

Jhanwar YS, Divgi C. 2005. Current status of therapy of solid tumors. The Journal of Nuclear Medicine 46:141S-150.

Jungerman JA, Yu K-HP, Zanelli CI. 1984. Radiation absorbed dose estimates at the cellular level for some electron-emitting radionuclides for radioimmunotherapy. International Journal of Applied Radiation and Isotopes 35:883-888.

Kassis AI. 2004. The amazing world of Auger electrons. International Journal of Radiation Biology 80:789-803.

Kassis AI, Adelstein SJ. 2005. Radiobiologic principles in radionuclide therapy. The Journal of Nuclear Medicine 46:4S-12.

Kim Y-K. 1972. Angular distributions of secondary electrons in the dipole approximation. Physical Review A 6:666-670.

LaVerne JA, Mozumder A. 1986. Effect of phase on the stopping and range distribution of low-energy electrons in water. Journal of Physical Chemistry 90:3242-3247.

Mariani G, Bodei L, Adelstein SJ, Kassis AI. 2000. Emerging roles for radiometabolic therapy of tumors based on Auger electron emission. The Journal of Nuclear Medicine 41:1519-1521.

Milenic DE, Brady ED, Brechbiel MW. 2004. Antibody-targeted radiation cancer therapy Nature Reviews Drug Discovery 3: 488-498.

Nahum AE. 1996. Microdosimetry and radiocurability: Modelling targeted therapy with $\beta$-emitters. Physics in Medicine and Biology 41:1957-1972.

Nikjoo H, Martin RF, Charlton DE, Terrissol M, Kandaiya S, Lobachevsky P. 1996. Modelling of Auger-induced DNA damage by incorporated I-125. Acta Oncologica 35:849-856.

Nikjoo $\mathrm{H}$, Terrissol $\mathrm{M}$, Hamm RN, Turner JE, Uehara S, Paretzke HG, Goodhead DT. 1994. Comparison of energy deposition in small cylindrical volumes by electrons generated by Monte Carlo track structure codes for gaseous and liquid water. Radiation Protection Dosimetry 52:165-169.

Nikjoo H, Uehara S, Emfietzoglou D, Cucinotta FA. 2006. Trackstructure codes in radiation research. Radiation Measurements 41:1052-1074.

O'Donoghue JA. 1999. Implications of nonuniform tumor doses for radioimmunotherapy. The Journal of Nuclear Medicine 40: 1337-1341.

Paretzke HG, Turner JE, Hamm RN, Wright HA, Ritchie RH. 1986. Calculated yields and fluctuations for electron degradation in liquid water and water vapour. Journal of Chemical Physics 84:3182-3188. 
Pomplun E. 1991. A new DNA target model for track structure calculations and its first application to I-125 Auger electrons. International Journal of Radiation Biology 59:625-642.

Seltzer SM. 1991. Electron-photon Monte Carlo calculations: The ETRAN code. Applied Radiation and Isotopes 42: 917-941.

Sharkey RM, Goldenberg DM. 2005. Perspectives on cancer therapy with radiolabeled monoclonal antibodies. The Journal of Nuclear Medicine 46:115S-127.

Siegel JA, Stabin MG. 1994. Absorbed fractions for electrons and beta particles in spheres of various sizes. The Journal of Nuclear Medicine 35:152-156.

Simpkin DJ, Mackie TR. 1990. EGS4 Monte Carlo determination of the beta dose kernel in water. Medical Physics 17:179-186.

Sgouros G. 1995. Radioimmunotherapy of micrometastasis: Sidestepping the solid-tumor hurdle. The Journal of Nuclear Medicine 36:1910-1912.

Spencer LV. 1955. Theory of electron penetration. Physical Review 98:1597-1615.

Stabin M. 2006. Nuclear medicine dosimetry. Physics in Medicine and Biology 51:R187-202.

Stabin M, Konijnenberg MW. 2000. Re-evaluation of absorbed fractions for photons and electrons in spheres of various sizes. The Journal of Nuclear Medicine 41:149-160.

Stewart RD, Wilson WE, McDonald JC, Strom DJ. 2002. Microdosimetric properties of ionizing electrons in water: A test of the PENELOPE code system. Physics in Medicine and Biology 47:79-88.
Syme AM, Kirkby C, Riauka TA, Fallone BG, McQuarrie SA. 2004. Monte Carlo investigation of single cell beta dosimetry for intraperitoneal radionuclide therapy. Physics in Medicine and Biology 49:1959-1972.

Torres-Garcia E, Garnica-Garza HM, Ferro-Flores G. 2006. Monte Carlo microdosimetry of ${ }^{188} \mathrm{Re}$ - and ${ }^{131}$ I-labelled antiCD20. Physics in Medicine and Biology 51:N349-356.

Uehara S, Nikjoo H, Goodhead DT. 1992. Cross-sections for water vapour for the Monte Carlo electron track structure code from $10 \mathrm{eV}$ to the $\mathrm{MeV}$ region. Physics in Medicine and Biology 37:1841-1858.

Uehara S, Nikjoo H, Goodhead DT. 1999. Comparison and assessment of electron cross sections for Monte Carlo track structure codes. Radiation Research 152:202-213.

Wheldon TE. 1994. Targeting radiation to tumours. International Journal of Radiation Biology 65:109-116.

Wheldon TE, O’Donoghue JA, Barett A, Michalowski AS. 1991. The curability of tumors of differing size by targeted radiotherapy using ${ }^{131} \mathrm{I}$ or ${ }^{90} \mathrm{Y}$. Radiotherapy and Oncology 21: 91-99.

Wilson WE, Nikjoo H. 1999. A Monte-Carlo code for positive ion track simulation. Radiation and Environmental Biophysics 38: 97-104.

Wright HA, Hamm RN, Turner JE, Howell RW, Rao DV, Sastry KSR. 1990. Calculations of physical and chemical reactions with DNA in aqueous solution from Auger cascades. Radiation Protection Dosimetry 31:59-62.

Zweit J. 1996. Radionuclides and carrier molecules for therapy. Physics in Medicine and Biology 41:1905-1914. 